\title{
Cardioprotective effects of traditional Chinese medicine Guanmaitong on acute myocardial infarction
}

\author{
XING-HUA WANG, GUANG-PING LI, WAN-SONG YANG, ZHAN-QUAN JIAO, HONG-MEI LIU and YAN-PING NI \\ Department of Cardiology, Tianjin Key Laboratory of Ionic-Molecular Function of Cardiovascular Disease, \\ Tianjin Institute of Cardiology, The Second Hospital of Tianjin Medical University, Tianjin 300211, P.R. China
}

Received January 30, 2015; Accepted February 19, 2016

DOI: $10.3892 /$ etm.2016.3888

\begin{abstract}
Guanmaitong (GMT) is a traditional Chinese herbal compound that has been used for the treatment of coronary heart disease (CHD) and other cardiovascular diseases. However, the efficacy of GMT in treating cardiovascular diseases remains unclear. The aim of the present study was to investigate the protective mechanisms and identify the targeted proteins and signaling networks associated with the physiological activity of GMT in a rat model of acute myocardial infarction (AMI). Sprague-Dawley rats were randomly allocated into five groups: Control group (sham-operated), the model group, and small, medium, and large dosage GMT groups. The rat model of AMI was established via ligation of the coronary artery. The results indicate that GMT was able to reduce myocardial infarction size and improve the activities of tumor necrosis factor- $\alpha$ (TNF- $\alpha$ ), intercellular adhesion molecule 1 (ICAM-1) and interleukin-1. Furthermore, the reduced apoptotic index of the GMT-treated cardiocytes ( $\mathrm{P}<0.05$ vs. model group) was in accordance with the downregulated expression of Bax and the upregulated expression of Bcl-2. In conclusion, GMT may exert a protective potential against myocardial infarction injury by inhibiting apoptosis and inflammation of cardiomyocytes, and may offer a promising adjunct treatment for CHD.
\end{abstract}

\section{Introduction}

Coronary heart disease (CHD), synonymously known as coronary artery disease (CAD) is the most predominant type of cardiovascular disease in developing countries (1). Acute myocardial infarction (AMI) is one of the most prevalent types of CHD (2), and may lead to an irreversible loss of

Correspondence to: Professor Guang-Ping Li, Department of Cardiology, Tianjin Key Laboratory of Ionic-Molecular Function of Cardiovascular Disease, Tianjin Institute of Cardiology, The Second Hospital of Tianjin Medical University, 23 Pingjiang Road, Tianjin 300211, P.R. China

E-mail: tjcardiol@126.com

Key words: guanmaitong, myocardial infarction, apoptosis, inflammation, Chinese medicine, bax cardiomyocytes and scar formation in the infarct area, which are major factors in the progression of heart failure $(3,4)$. Therefore, investigation of novel treatments to improve the prognosis of AMI is an area of intense activity.

Prior studies have reported the effects of various herb-derived compounds on clinical symptoms, biomarkers and mortality in AMI patients and animal models (5-7). However, the mechanisms underlying these effects require further investigation and systematic review.

The use of a combination of multiple herbs is designed to exploit the additive or synergistic activities of individual herbs, as well as to balance or neutralize the toxic effects of certain herbal components by others in the mixture (8). Guanmaitong (GMT) consists primarily of the herbs Salvia miltiorrhiza, Safflower (Carthamus tinctorius) and Polygonum multiflorum, has been applied to the treatment of CHDs such as angina pectoris and myocardial ischemia in China (9). The three medicinal herbs are commonly used in traditional Chinese medicine and have previously been shown to be physiologically active in human vascular endothelial cells (10). Tetrahydroxystilbene-glucoside, one of the active ingredients of F. multiflorum, has been reported to exert a protective effect on the cardiovascular system by influencing cellular antioxidant capacity and inhibiting doxorubicin-induced apoptosis in cardiomyocytes $(11,12)$. The pharmacological effects of S. miltiorrhiza extracts include antioxidative, anti-apoptotic and vasodilatory activities, which may be affected by the inhibition of intercellular adhesion molecule 1 (ICAM-1) expression to protect endothelial function and inhibit atherogenesis, and promoting the role of $S$. miltiorrhiza in cardiovascular and cerebrovascular systems (13-15). In previous studies, Safflower has been demonstrated to reduce cardiovascular disease risk in rats $(16,17)$.

The aim of the present study was investigate the cardioprotective effects of GMT and to elucidate possible mechanisms underlying its effect on myocardial apoptosis and inflammatory response in rats with $\mathrm{AMI}$.

\section{Materials and methods}

Animals. A total of 60 healthy adult male Sprague-Dawley (SD) rats, aged 6-8 weeks and weighing 220-250 g, were provided by the Experimental Animal Center of PLA Academy of Military Medical Sciences (Beijing, China) and acclimated for at least 
three days [license no. SCXK (Army) 2007-004]. All animals were housed in separated cages with laboratory chow and tap water ad libitum. All animal experiments were performed in accordance with the National Institute of Health Guide for the Care and Use of Laboratory Animals, and experiments were approved by the University Laboratory Animal Research Committee of Tianjin Medical University (Tianjin, China).

Experimental design and protocol. SD rats were randomly allocated into five equal groups ( $\mathrm{n}=12$ /group): Sham-operated control group (S), model group (M), and small $(0.55 \mathrm{~g} / \mathrm{kg} / \mathrm{day}$; GL), medium (1.1 g/kg/day; GM), and large $(2.2 \mathrm{~g} / \mathrm{kg} / \mathrm{day}$; GH) dosage GMT groups. GMT was provided by Tianjin Tongrentang Group Co., Ltd. (Tianjin, China). Control and model groups received an equal quantity of vehicle. After 14 days of treatment, animals underwent AMI surgery.

Animal model establishment. An AMI model was established in rats by ligation of the left anterior descending coronary artery for $24 \mathrm{~h}$. The surgical procedure was performed according to a previous study (18), with minor modifications. Briefly, SD rats were anesthetized with $10 \%$ chloral hydrate $(0.3 \mathrm{ml} / 100 \mathrm{~g}$, intraperitoneally; Sigma-Aldrich, St. Louis, MO, USA), then a left thoracotomy was performed. The incised area was extended using forceps and the pericardium was opened. Following tracheal intubation, the rats were ventilated using a respirator (ALC-V8; Alcott Biotech Co., Ltd., Shanghai, China) with room air at a tidal volume of $25 \mathrm{ml} / \mathrm{min}$ and a respiratory rate of 70 breaths $/ \mathrm{min}$. The heart was exteriorized and ligated at the proximal left anterior descending coronary artery $2-3 \mathrm{~mm}$ from its origin between the pulmonary artery conus and the left atrium using a 5-0 Prolene suture (WEGO Inc., Shandong, China). The heart was returned to its normal position and the thorax was closed. Sham-operated rats underwent an identical surgical procedure as described above except that the suture was not tightened around the coronary artery.

Measurement of myocardial infarct size and histological analysis. A 2,3,5-triphenyltetrazolium chloride (TTC) assay was used to determine myocardial infarct size. TTC was provided by Amresco (Amresco LLC, Solon, OH, USA). In brief, the heart was transversely cut across the left ventricle, and sections $2-3 \mathrm{~mm}$ thick were incubated in $0.5 \%$ TTC solution prepared in phosphate buffer ( $\mathrm{pH}$ 7.4; Sangon Biotech Co., Ltd., Shanghai, China) for $30 \mathrm{~min}$ at $37^{\circ} \mathrm{C}$, following which they were fixed with $10 \%$ formalin (Sangon Biotech Co., Ltd.). Non-ischemic and viable ischemic myocardium were stained red, while the infarcted myocardium appeared pale grey or white. For histological analysis, $5-\mu \mathrm{m}$ sections from the left ventricle were stained with hematoxylin and eosin (HE; Beijing Zhongshan Golden Bridge Biotechnology Co., Ltd., Beijing, China). The pathological features were observed using a microscope (BX53; Olympus Corporation, Tokyo, Japan) at a magnification of $\mathrm{x} 400$.

Measurement of cardiac marker enzyme activity. Abdominal aortic blood samples $(4 \mathrm{ml})$ was separated by centrifugation at $840 \mathrm{xg}$ for $10 \mathrm{~min}$ at $4^{\circ} \mathrm{C}$. The activities of serum creatine kinase (CK; 812060103), creatine kinase-MB (CK-MB; 812060202), and lactate dehydrogenase (LDH; 812060403)
Table I. Polymerase chain reaction primer sequences.

\begin{tabular}{ll} 
Gene & \multicolumn{1}{c}{ Sequence (5'-3') } \\
\hline IL-1 & F, AAGACAAGCCTGTGTTGCTGAAGG \\
& R,TCCCAGAAGAAAATGAGGTCGGTC \\
TNF- $\alpha$ & F, AAATGGGCTCCCTCTCATCAGTTC \\
& R, TCTGCTTGGTGGTTTGGCTACGAC \\
ICAM-1 & F, GGGTTGGAGACTAACTGGA \\
& R, GCACCGCAGGATGAGGTTCTT \\
GAPDH & F, AACGACCCCTCATTGACCT \\
& R, CCCCATTTGATGTTAGCGGG
\end{tabular}

$\mathrm{F}$, forward; R, reverse; IL-1, interleukin-1; TNF- $\alpha$, tumor necrosis factor- $\alpha$; ICAM-1, intercellular adhesion molecule 1 .

were measured spectrophotometrically according to the specifications of commercial diagnostic kits (Shanghai Kehua Medical Instruments Co., Ltd., Shanghai, China).

Reverse transcription-quantitative polymerase chain reaction (RT-qPCR) analysis. Rats were sacrificed with $10 \%$ chloral hydrate $(2 \mathrm{ml} / 100 \mathrm{~g})$ and total RNA was extracted from the rat heart tissues using TRIzol (Invitrogen; Thermo Fisher Scientific, Inc., Waltham, MA, USA) according to the manufacturer's instructions. RNA yields and purity were assessed by spectrophotometric analysis (BioPhotometer Plus; Eppendorf, Shanghai, China) at 260 and $280 \mathrm{~nm}$. Total RNA $(1 \mu \mathrm{g})$ from each well was subjected to reverse transcription with oligo dT (19) primers, dNTPs (both Takara Bio, Inc., Otsu, Japan) and M-MLV reverse transcriptase (Promega Corporation, Madison, WI, USA) in a total reaction volume of $20 \mu \mathrm{l}$. Following DNase treatment (Sigma-Aldrich), the 20- $\mu$ l RT-qPCR reaction system consisted of $10 \mu 1$ SYBR Green Mix (Takara Bio, Inc.), $0.5 \mu 1$ of each primer (Table I) (Invitrogen), $1 \mu \mathrm{l} \mathrm{cDNA}$ and $8 \mu \mathrm{l}$ double-distilled water. RT-qPCR was performed using an ABI 7300 Real-Time PCR System (Applied Biosystems; Thermo Fisher Scientific, Inc.) and data were analyzed using the accompanying ABI 7300 software. Thermal cycling conditions were as follows: Initial denaturation at $95^{\circ} \mathrm{C}$ for $5 \mathrm{~min} ; 40$ cycles at $95^{\circ} \mathrm{C}$ for $30 \mathrm{sec}$ and $58^{\circ} \mathrm{C}$ for $30 \mathrm{sec}$; elongation at $72^{\circ} \mathrm{C}$ for $30 \mathrm{sec}$; a final cycle at $95^{\circ} \mathrm{C}$ for $15 \mathrm{sec}$ and $60^{\circ} \mathrm{C}$ for $15 \mathrm{sec}$; followed by dissociation at $95^{\circ} \mathrm{C}$ for $15 \mathrm{sec}$. All values obtained with the tumor necrosis factor- $\alpha$ (TNF- $\alpha$ ), interleukin (IL-1) or ICAM-1 primers were normalized against the values obtained with the GAPDH primers, according to the $2^{-\Delta \Delta \mathrm{Cq}}$ method (20). The results are expressed as the relative integrated intensity. Negative controls (no cDNA) and RT controls (no reverse transcriptase) were performed. All reactions were performed in triplicate.

Enzyme-linked immunosorbent assay (ELISA) detection of $I L-1$. ELISA measurements of IL-1 expression were performed in duplicate using a specific, commercially available IL-1 ELISA kit (Cusabio Biotech Co., Ltd., Wuhan, China) in accordance with the manufacturer's instructions, and analyzed using an ELISA reader (Tecan Trading AG, Männedorf, Switzerland) at $450 \mathrm{~nm}$. 
Table II. Effect of Guanmaitong on myocardial infarct tissue size.

\begin{tabular}{lccc}
\hline Group & Ventricle weight $(\mathrm{g})$ & Infarction weight $(\mathrm{g})$ & Infarction rate $(\%)$ \\
\hline Model & $0.61 \pm 0.08$ & $0.19 \pm 0.02$ & $30.02 \pm 4.32^{\mathrm{a}}$ \\
Sham & $0.57 \pm 0.06$ & - & - \\
Low dosage & $0.62 \pm 0.05$ & $0.15 \pm 0.05$ & $23.16 \pm 4.01^{\mathrm{b}}$ \\
Medium dosage & $0.65 \pm 0.09$ & $0.11 \pm 0.03$ & $17.83 \pm 2.37^{\mathrm{b}}$ \\
High dosage & $0.63 \pm 0.04$ & $0.09 \pm 0.04$ & $15.71 \pm 3.32^{\mathrm{b}}$ \\
\hline
\end{tabular}

Data presented as the mean \pm standard deviation. ${ }^{\mathrm{a}} \mathrm{P}<0.05$ vs. sham group; ${ }^{\text {b }}<0.05$ vs. model group.
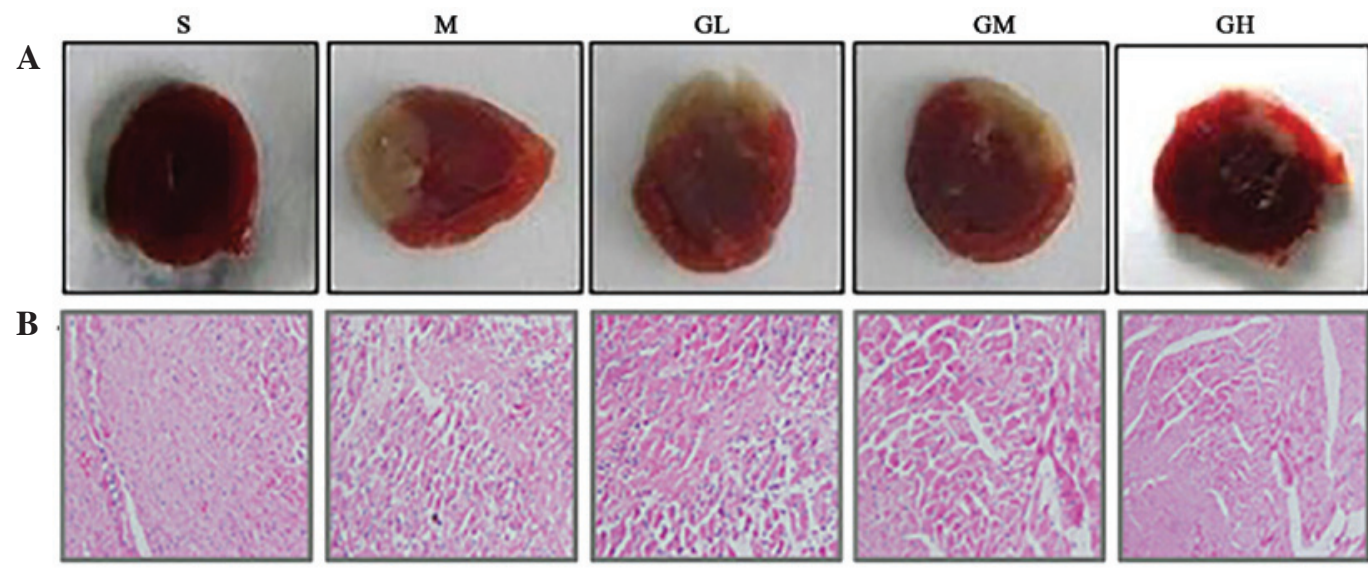

Figure 1. Myocardial infarct size and hematoxylin and eosin (HE) staining. (A) Normal myocardium is stained red, while pale grey areas indicate infarct areas. (B) HE staining was conducted to detect the pathological alterations (magnification, x400). S, sham group; M, model group; GL, low dosage group; GM, medium dosage group; GH, high dosage group.

Western blot analysis. Myocardial tissue (100 mg) was grinded in liquid nitrogen and incubated with radioimmunoprecipitation assay lysis buffer, containing $20 \mathrm{mM}$ HEPES, $0.5 \%$ NP-40 (both Sigma-Aldrich), $1 \%$ protease inhibitor cocktail (Promega Corporation), $200 \mathrm{mM} \mathrm{KCl,} \mathrm{20 \%} \mathrm{glyc-}$ erol and 0.5 mM EDTA (all Sangon Biotech Co., Ltd.), to extract total protein. Subsequently, $30 \mu \mathrm{g}$ protein/lane was subjected to $10 \%$ SDS-PAGE and transferred onto nitrocellulose membranes (EMD Millipore, Billerica, MA, USA). Membranes were blocked with $5 \%$ bovine serum albumin and subsequently incubated with antibodies against B-cell lymphoma 2 (Bcl-2; 1:1,000; 2876), Bcl-2-associated X protein (Bax; 1:1,000; 2772), TNF- $\alpha$ (1:2,000; MAB510) ICAM-1 $(1: 1,000 ; \mathrm{ab} 124760)$ and GAPDH (1:2,000; AB-M-M001), as the internal control, at $4^{\circ} \mathrm{C}$ overnight. Following washing six times for 30 min with Tris-buffered saline with Tween 20 (TBST), the membranes were incubated with horseradish peroxidase (HRP)-conjugated goat anti-rabbit IgG (7074) or horse anti-mouse IgG (7076) secondary antibodies (both 1:3,000; Cell Signaling Technology, Inc., Danvers, MA, USA) for $1 \mathrm{~h}$ at room temperature to detect the primary antibody. Following further washing with TBST for $30 \mathrm{~min}$, the intensity of immunoreactive bands was estimated using an imaging densitometer (Gene Tools 3.06; Gene Company Ltd., Hong Kong). Rabbit polyclonal anti-Bax and anti-Bcl-2 antibodies were purchased from Cell Signaling Technology, Inc., monoclonal anti-TNF- $\alpha$ antibody from R\&D Systems
(Minneapolis, MN, USA), rabbit polyclonal anti-ICAM-1 antibody from Abcam (Cambridge, MA, USA) and anti-GAPDH antibody (1:2,000; AB-M-M001) from Hangzhou Xianzhi Biotechnology (Hangzhou, China).

Immunohistochemical detection of Bcl-2 and Bax expression. Tissues were conventionally fixed with $10 \%$ formalin, then dehydrated with alcohol, embedded with paraffin wax and continuously sectioned at $5 \mu \mathrm{m}$. Sections were incubated overnight at $4^{\circ} \mathrm{C}$ with primary anti-Bcl-2 (BA0412) and anti-Bax (BA0315) antibodies (both 1:500; Wuhan Boster Biological Technology Ltd., Wuhan, China). Negative control were performed which involved the omission of primary antibody and use of phosphate-buffered saline (PBS). Sections were then rinsed with PBS and incubated for $1 \mathrm{~h}$ with HRP-conjugated secondary antibody (1:2,000; ZB2301; Beijing Zhongshan Golden Bridge Biotechnology Co., Ltd.). The reaction was visualized using a solution of 3,3'-diaminobenzidine (Beijing Zhongshan Golden Bridge Biotechnology Co., Ltd.). For quantification, the integral optical density of Bax and Bcl-2 staining were calculated using Image-Pro Plus 6.0 software (Media Cybernetics, Inc., Rockville, MD, USA).

Statistical analysis. Data are reported as the mean \pm standard deviation. Statistical significance was determined using one-way analysis of variance tests followed by Dunnett's test. Statistical analyses were performed using the software 
Table III. Effect of Guanmaitong on cell apoptosis.

\begin{tabular}{lccc}
\hline Group & Apoptosis index $(\%)$ & $\eta(\mathrm{Bcl}-2) \%$ & $\eta(\mathrm{Bax}) \%$ \\
\hline Model & $31.8 \pm 1.9^{\mathrm{a}}$ & $13.1 \pm 1.3^{\mathrm{a}}$ & $52.7 \pm 2.0^{\mathrm{a}}$ \\
Sham & $2.3 \pm 0.9$ & $11.4 \pm 0.9$ & $13.3 \pm 1.0$ \\
Low dosage & $25.1 \pm 2.1^{\mathrm{b}}$ & $15.3 \pm 1.0$ & $49.5 \pm 5.1$ \\
Medium dosage & $23.2 \pm 1.6^{\mathrm{b}}$ & $18.6 \pm 1.8^{\mathrm{a}}$ & $45.1 \pm 2.7^{\mathrm{b}}$ \\
High dosage & $18.5 \pm 5.1^{\mathrm{b}}$ & $21.7 \pm 2.2^{\mathrm{a}}$ & $34.4 \pm 3.3^{\mathrm{b}}$ \\
\hline
\end{tabular}

$\eta \%$ indicates the positive area/total area. ${ }^{\mathrm{a}} \mathrm{P}<0.05$ vs. sham group; ${ }^{\mathrm{b}} \mathrm{P}<0.05$ vs. model group, as determined by one-way analysis of variance followed by Dunnett's test. Bcl-2, B-cell lymphoma 2; Bax, Bcl-2-associated X protein.

package StatView 5.0J (SAS Institute, Cary, NC, USA). $\mathrm{P}<0.05$ was considered to indicate a statistically significant difference.

\section{Results}

GMT reduces infarct area and pathological changes in cellular morphology. Representative illustrations of infarction tissue size (pale grey areas) as stained by TTC are shown in Fig. 1A. Sham-operated rats exhibited no evidence of infarcted tissue in the left ventricle. The model group presented with a large unstained area $(30.02 \pm 4.32 \% \mathrm{vs}$. sham group; Table II). The infarction size significantly reduced in GMT groups compared with the model group, and the size was smallest in the high dosage group $(15.71 \pm 3.32 \%)$ compared with the medium $(17.83 \pm 2.37 \%)$ and small $(23.16 \pm 4.01 \%)$ dosage groups. Regarding HE staining, the model group showed augmentation and loose arrangement of the myocardial fibers, staining asymmetry, deformity of the nucleus and marked edema and infiltration. By contrast, tissues from the SD rats treated with GMT exhibited markedly improved pathological changes compared with model group (Fig. 1B).

Effects of GMT on levels of cardiac marker enzyme activity. Fig. 2 shows that compared with the sham-operated group, the serum CK, CK-MB and LDH activities in the model group increased significantly $(\mathrm{P}<0.05)$. Treatment with GMT attenuated these elevations, and the various GMT dosages produced varying degrees of reduction (Fig. 2).

Effect of GMT on cardiomyocyte apoptosis. Adult cardiomyocytes are post-mitotic cells, and thus have a limited response capability to damage (21). Prior studies have suggested that apoptosis is increased in acute and chronic heart pathologies, were the process appears to be crucially involved (21). To clarify the possible mechanism underlying anti-apoptotic effect exerted by GMT, two key factors of intrinsic pathway, Bcl-2 and Bax, in the infarct heart tissues. Using western blot and immunohistochemical assays (Fig. 3), a significant reduction in Bcl-2 expression and an increase in Bax expression was observed in the cardiocytes of the model group, as compared with the sham-operated group. Treatment with GMT resulted

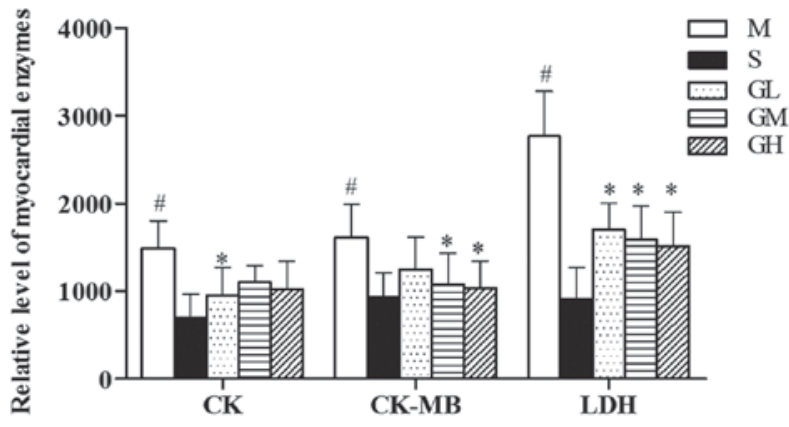

Figure 2. Effect of Guanmaitong (GMT) on cardiac marker enzyme activity in rats. Treatment with GMT attenuated the elevation of cardiac marker enzyme activity, and different dose groups of GMT decline to different extent. ${ }^{\text {P }}<0.05$ vs. sham group; ${ }^{*} \mathrm{P}<0.05$ vs. model group, as determined by one-way analysis of variance followed by Dunnett's test. M, model group; $\mathrm{S}$, sham group; GL, low dosage group; GM, medium dosage group; GH, high dosage group; CK, creatine kinase; $\mathrm{LDH}$, lactate dehydrogenase.

in enhanced Bcl-2 expression, reduced Bax expression and attenuated the ratio of $\mathrm{Bcl}-2 / \mathrm{Bax}(\mathrm{P}<0.05)$ (Table III). Furthermore, the higher dosage groups (1.1 and $2.2 \mathrm{~g} / \mathrm{kg} / \mathrm{day})$ exhibited more marked differences compared with the low dosage group ( $0.55 \mathrm{~g} / \mathrm{kg} / \mathrm{day})$.

Expression of inflammation-related cytokine mRNAs in $A M I$ via $R T$-qPCR assay. At $24 \mathrm{~h}$ after AMI induction the mRNA expression levels of IL-1, TNF- $\alpha$ and ICAM-1 in the model group significantly increased $(\mathrm{P}<0.05$ vs. sham group; Fig. 4), and a reversed trend was observed in GMT groups. In particular, the mRNA expression levels of TNF- $\alpha$ and ICAM-1 were significantly reduced in all three GMT-treated groups ( $\mathrm{P}<0.05$ vs. model group), and were dosage-correlated. The expression levels of IL-1 mRNA were significantly reduced in the medium and high dosage groups $(\mathrm{P}<0.05$ vs. model group), whereas no significant difference was detected in the low dosage group.

Effect of GMT on protein expression levels of TNF- $\alpha$, ICAM-1 and $I L-1$. The western blot analysis results indicated that ICAM-1 expression increased in the model group (vs. sham group) and decreased significantly in the medium and high dosage GMT groups ( $\mathrm{P}<0.05$ vs. model group; Fig. 5A). By contrast, a statistically significant elevation in $\mathrm{TNF}-\alpha$ protein expression was detected in all three GMT groups $(\mathrm{P}<0.05$ vs. model group; Fig. 5B). The results of the ELISA assay indicate that the protein expression levels of IL-1 increased significantly in the model group ( $\mathrm{P}<0.05$ vs. sham group), and that this elevation was significantly inhibited in all three GMT groups ( $\mathrm{P}<0.05$ vs. model group).

\section{Discussion}

Apoptosis, the physiological process of programmed cell death, may contribute to various cardiac disorders (22). Apoptosis has been reported to contribute to the loss of cardiomyocytes and is recognized as a predictor of adverse outcomes in patients with cardiac diseases or heart failure (23). Consequently, the interruption of apoptotic pathways may facilitate the development of novel strategies to reverse or attenuate heart 


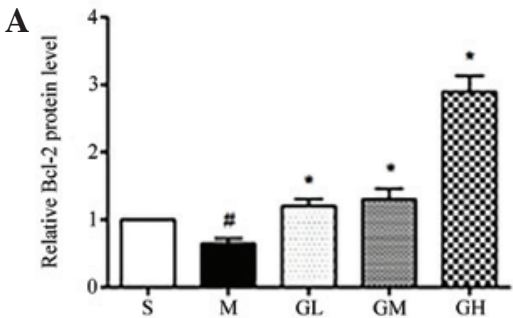

B
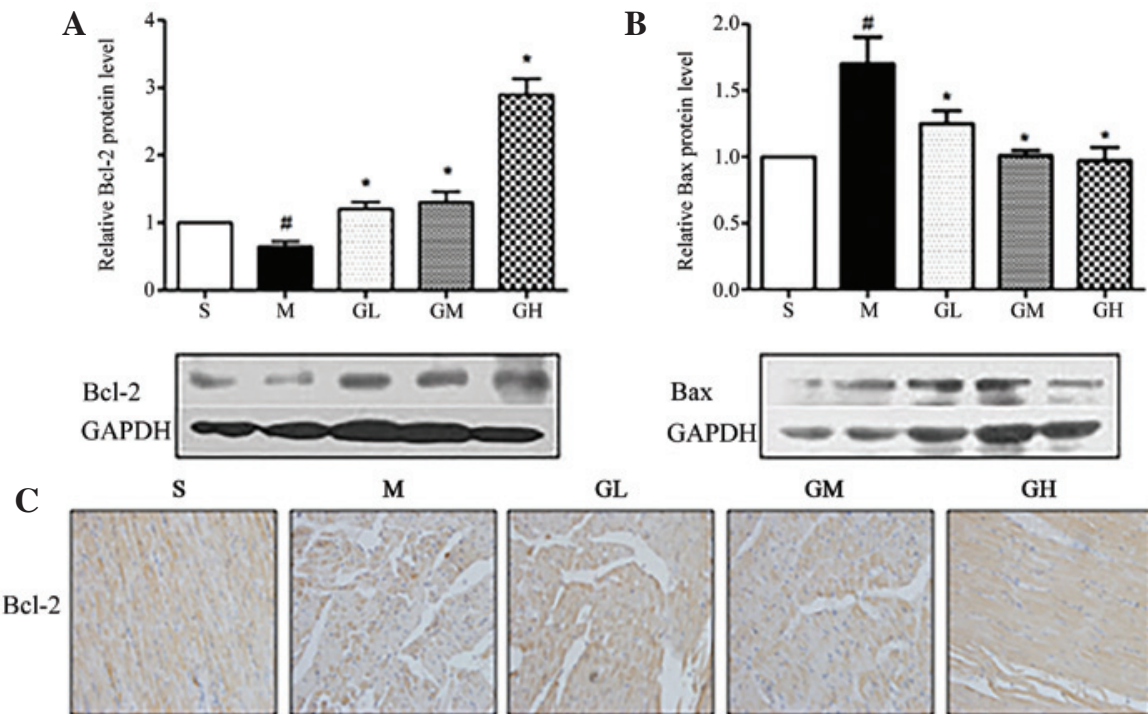

GL

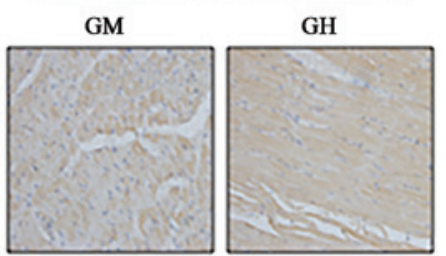

Bax
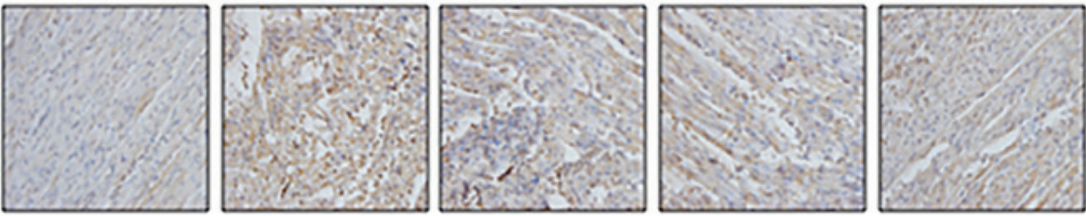

Figure 3. Expression of Bcl-2 and Bax in GMT-treated Sprague-Dawley rats with acute myocardial infarction. (A and B) Western Blot was used to evaluate the protein expression of Bcl-2 and Bax. (C) Immunohistochemical analysis was used to detected the expression of Bcl-2 and Bax (magnification, $\mathrm{x} 400)$. ${ }^{.} \mathrm{P}<0.05$ vs. sham group; " $\mathrm{P}<0.05$ vs. model group, as determined by one-way analysis of variance followed by Dunnett's test. S, sham group; M, model group; GL, low dosage group; GM, medium dosage group; GH, high dosage group; Bcl-2, B-cell lymphoma 2; Bax, Bcl-2-associated X protein.

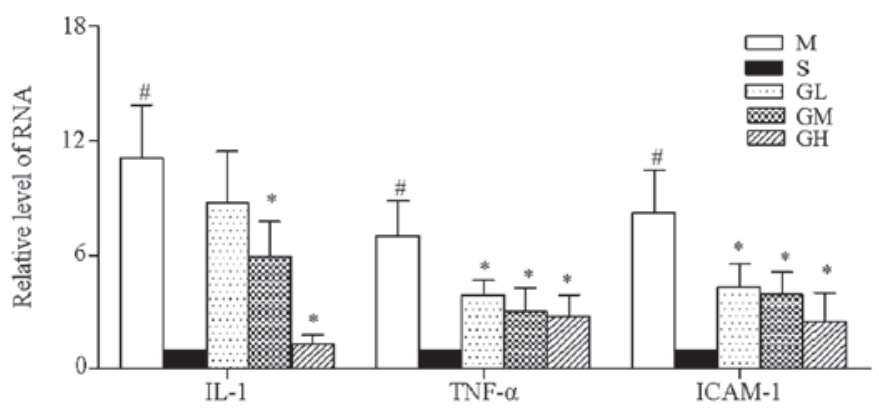

Figure 4. Detection of inflammation-related cytokine expression using reverse transcription-quantitative polymerase chain reaction. ${ }^{*} \mathrm{P}<0.05$ vs. sham group; ${ }^{*} \mathrm{P}<0.05$ vs. model group, as determined by one-way analysis of variance followed by Dunnett's test. M, model group; S, sham group; GL, low dosage group; GM, medium dosage group; GH, high dosage group; IL-1, interleukin-1; TNF- $\alpha$, tumor necrosis factor- $\alpha$; ICAM-1, intercellular adhesion molecule 1.

A

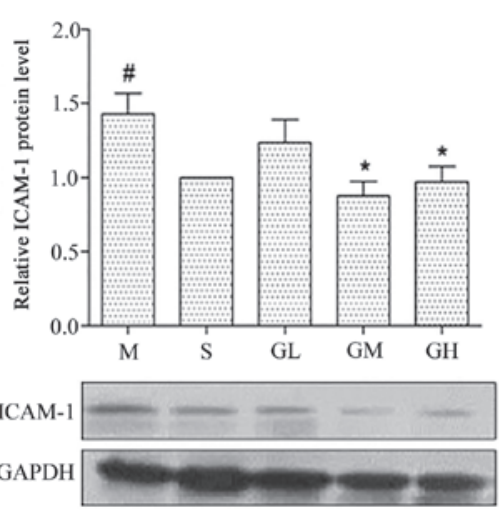

B
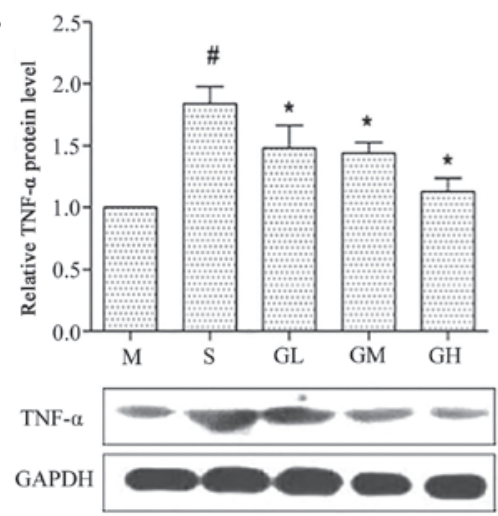

C

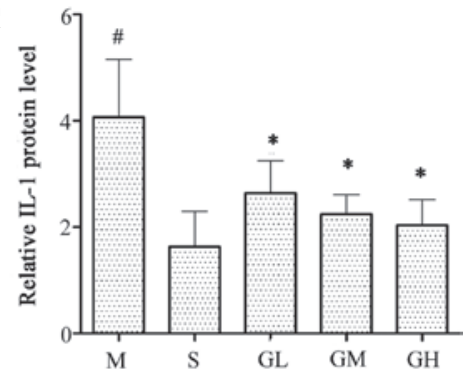

Figure 5. Detection of inflammation-related cytokine proteins in Guanmaitong-treated Sprague-Dawley rats with acute myocardial infarction. (A and B) Western blot was used to analyze the protein expression levels of ICAM-1 and TNF- $\alpha$. (C) IL-1 expression was analyzed using an ELISA assay. "P<0.05 vs. sham group; ${ }^{*} \mathrm{P}<0.05$ vs. model group, as determined by one-way analysis of variance followed by Dunnett's test. M, model group; S, sham group; GL, low dosage group; GM, medium dosage group; GH, high dosage group; ICAM-1, intercellular adhesion molecule 1 ; TNF- $\alpha$, tumor necrosis factor- $\alpha$; IL-1, interleukin-1. 
failure (24,25). Certain active components of GMT have been reported to have anti-apoptotic effects, and are used as a classic prescription in traditional Chinese medicine for the treatment of cardiovascular diseases (12-15). Therefore, it was speculated for the purposes of the present study that GMT could salvage these cardiocytes and prevent ischemic cell loss induced by apoptosis. In the present study, GMT treatment upregulated the expression of the anti-apoptotic protein, $\mathrm{Bcl}-2$, and downregulated the expression of the proapoptotic protein, Bax. Upregulation of Bcl-2 enhanced the formation of heterodimers with Bax, resulting in fewer available Bax proteins for the formation of homodimers. It is well known that if Bax homodimers predominate cell death will occur $(26,27)$.

AMI is currently speculated to involve the process of inflammation, which is a hallmark throughout the distinct stages of atherosclerosis and plaque rupture (28). TNF- $\alpha$ is a key inflammatory cytokine which exerts pleiotropic biological effects and is crucially involved in cardiovascular diseases such as AMI (29). The inflammatory reaction caused by TNF- $\alpha$ is able to induce upregulation of IL-1 and ICAM-1; however, the increased expression of TNF- $\alpha$ may also regulate apoptosis by inhibiting the expression levels of the anti-apoptosis factor Bcl-2 (30). Another inflammatory cytokine, IL-1, has been proposed as a crucial mediator in the inflammatory response and AMI. In patients with ST-segment elevation AMI, IL-1 blockade with the IL-1 receptor antagonist anakinra is safe and ameliorates left ventricular remodeling, and can significantly increase and induce the expression of ICAM-1 in ischemic heart disease $(31,32)$. ICAM-1 belongs to the super-family of immunoglobulin-like adhesion molecules and is critical for various physiological and pathological processes (33). It has previously been shown that ICAM-1 is able to induce and aggravate AMI, and its expression levels are closely associated with the extent of myocardial damage (34).

In the present study, the expression of a number of inflammatory cytokines increased rapidly in model group, while GMT treatment effectively reversed this change by influencing the expression of these factors and apoptosis regulators. Therefore, the present data support the cardioprotective capacity of GMT and suggest possible mechanisms underlying the observed anti-inflammatory and anti-apoptosis effects. Further mechanistic studies aimed at identifying the detailed signaling pathways upstream of TNF- $\alpha$ and $\mathrm{Bcl}-2$ are required, in order to elucidate the molecular mechanisms underlying GMT and provide a theoretical basis for its clinical application.

\section{Acknowledgements}

This study was supported by grants from the Second Hospital of Tianjin Medical University (grant no. y1106) and Tianjin Tongrentang Group Co., Ltd. (Tianjin, China).

\section{References}

1. Pranavchand R and Reddy B: Current status of understanding of the genetic etiology of coronary heart disease. J Postgrad Med 59: 30-41, 2013.

2. Li C, Pei F, Zhu X, Duan DD and Zeng C: Circulating microRNAs as novel and sensitive biomarkers of acute myocardial infarction. Clin Biochem 45: 727-732, 2012.
3. Frangogiannis NG: The immune system and the remodeling infarcted heart: Cell biological insights and therapeutic opportunities. J Cardiovasc Pharmacol 63: 185-195, 2014.

4. Dauwe DF and Janssens SP: Stem cell therapy for the treatment of myocardial infarction. Curr Pharm Des 17: 3328-3340, 2011.

5. Liang X, Chen X, Liang Q, Zhang H, Hu P, Wang Y and Luo G: Metabonomic study of Chinese medicine Shuanglong formula as an effective treatment for myocardial infarction in rats. J Proteome Res 10: 790-799, 2010

6. Wang Y, Qian P, Liu P, Wei L, Cao M, Zhou L, Zhou D and Lin ZX: Effects of Panax notoginseng flower extract on the TGF- $\beta /$ Smad signal transduction pathway in heart remodeling of human chymase transgenic mice. Mol Med Rep 5: 1443-1448, 2012.

7. Chen $\mathrm{KJ}$ and $\mathrm{Xu} \mathrm{H}$ : The integration of traditional Chinese medicine and Western medicine. European Review 11: 225-235, 2003.

8. Zhu YP and Woerdenbag HJ: Traditional Chinese herbal medicine. Pharmacy World and Science 17: 103-112, 1995.

9. Pan SY, Chen SB, Dong HG, Yu ZL, Dong JC, Long ZX, Fong WF, Han YF and Ko KM: New perspectives on Chinese herbal medicine (Zhong-Yao) research and development. Evid Based Complement Alternat Med 2011: 403709, 2011.

10. Ling S, Nheu L, Dai A, Guo Z and Komesaroff P: Effects of four medicinal herbs on human vascular endothelial cells in culture. Int J Cardiol 128: 350-358, 2008.

11. Hong CY, Lo YC, Tan FC, Wei YH and Chen CF: Astrafalus membranaceus and Polygonum multiflourm protect rat heart mitochondria against lipid peroxidation. Am J Chin Med 22: 63-70, 1994.

12. Zhang SH, Wang WQ and Wang JL: Protective effect of tetrahydroxystilbene glucoside on cardiotoxicity induced by doxorubicin in vitro and in vivo. Acta Pharmacol Sin 30: 1479-1487, 2009.

13. Zheng CS, Xu XJ, Ye HZ, Wu GW, Xu HF, Li XH, Huang SP and Liu XX: Computational pharmacological comparison of Salvia miltiorrhiza and Panax notoginseng used in the therapy of cardiovascular diseases. Exp Ther Med 6: 1163-1168, 2013.

14. Fong CC, Wei F, Chen Y, Yu WK, Koon CM, Leung PC, Fung KP, Lau CB and Yang M: Danshen-Gegen decoction exerts proliferative effect on rat cardiac myoblasts H9c2 via MAPK and insulin pathways. J Ethnopharmacol 138: 60-66, 2011.

15. Wing-Shing Cheung D, Koon CM, Ng CF, Leung PC, Fung KP, Kar-Sing Poon S and Bik-San Lau C: The roots of Salvia miltiorrhiza (Danshen) and Pueraria lobata (Gegen) inhibit atherogenic events: A study of the combination effects of the 2-herb formula. J Ethnopharmacol 143: 859-866, 2012.

16. Wan LH, Chen J, Li L, Xiong WB and Zhou LM: Protective effects of Carthamus tinctorius injection on isoprenaline-induced myocardial injury in rats. Pharm Biol 49: 1204-1209, 2011.

17. Han SY, Li HX, Ma X, Zhang K, Ma ZZ, Jiang Y and Tu PF: Evaluation of the anti-myocardial ischemia effect of individual and combined extracts of Panax notoginseng and Carthamus tinctorius in rats. Ethnopharmacol 145: 722-727, 2013.

18. Maclean D, Fishbein MC, Braunwald E and Maroko PR: Long-term preservation of ischemic myocardium after experimentalcoronary artery occlusion. J Clin Invest 61: 541-551, 1978.

19. Whelan RS, Kaplinskiy V and Kitsis RN: Cell death in the pathogenesis of heart disease: mechanisms and significance. Annu Rev Physio 72: 19-44, 2010.

20. Livak KJ and Schmittgen TD: Analysis of relative gene expression data using real-time quantitative PCR and the $2^{-\Delta \Delta C t}$ method. Methods 25: 402-408, 2001.

21. Favaloro B, Allocati N, Graziano V, Di Ilio C and De Laurenzi V: Role of apoptosis in disease. Aging (Albany NY) 4: 330-349, 2012.

22. Lee SD, Chu CH, Huang EJ, Lu MC, Liu JY, Liu CJ, Hsu HH, Lin JA, Kuo WW and Huang CY: Roles of insulin-like growth factor II in cardiomyoblast apoptosis and in hypertensive rat heart with abdominal aorta ligation. Am J Physiol Endocrinol Metab 291: E306-E314, 2006.

23. Liou CM, Tsai SC, Kuo $\mathrm{CH}$, Ting $\mathrm{H}$ and Lee SD: Cardiac Fas-dependent and mitochondria-dependent apoptosis after chronic cocaine abuse. Int J Mol Sci 15: 5988-6001, 2014.

24. Sinha-Hikim I, Shen R, Nzenwa I, Gelfand R, Mahata SK and Sinha-Hikim AP: Minocycline suppresses oxidative stress and attenuates fetal cardiac myocyte apoptosis triggered by in utero cocaine exposure. Apoptosis 16: 563-573, 2011.

25. Gill C, Mestril R and Samali A: Losing heart: The role of apoptosis in heart disease-a novel therapeutic target? FASEB J 16: 135-146, 2002. 
26. Bansal N, Marchion DC, Bicaku E, Xiong Y, Chen N, Stickles XB Sawah EA, Wenham RM, Apte SM, Gonzalez-Bosquet J, et al: BCL2 antagonist of cell death kinases, phosphatases and ovarian cancer sensitivity to cisplatin. J Gynecol Oncol 23: 35-42, 2012.

27. Xu Z, Dong Y, Wu X, Zhang J, McAuliffe S, Pan C, Zhang Y, Ichinose F, Yue Y and Xie Z: The potential dual effects of anesthetic isoflurane on A $\beta$-induced apoptosis. Curr Alzheimer Res 8: 741-752, 2011.

28. Lin XM, Zhang ZY, Wang LF, Zhang L, Liu Y, Liu XL, Yang XC, Cui $L$ and Zhang L: Attenuation of tumor necrosis factor-alpha elevation and improved heart function by postconditioning for 60 seconds in patients with acute myocardial infarction. Chin Med J (Engl) 123: 1833-1839, 2010.

29. Tuttolomondo A, Di Raimondo D, Pecoraro R, Arnao V, Pinto A and Licata G: Inflammation in ischemic stroke subtypes. Curr Pharm Des 18: 4289-4310, 2012.

30. Chen M, Quintans J, Fuks Z, Thompson C, Kufe DW and Weichselbaum RR: Suppression of Bcl-2 messenger RNA production may mediate apoptosis after ionizing radiation, tumor necrosis factor and ceramide. Cancer Res 55: 991-994, 1995.
31. Arnson Y: Circulating levels of adhesion molecules ICAM-1, VCAM-1, E-Selectin, VEGF and their associations with age and troponin in patients with acute coronary syndrome. J Am Coll Cardiol 63: A177, 2014.

32. Abbate A, Kontos MC, Grizzard JD, Biondi-Zoccai GG, Van Tassell BW, Robati R, Roach LM, Arena RA, Roberts CS, Varma A, et al; VCU-ART Investigators: Interleukin-1 blockade with anakinra to prevent adverse cardiac remodeling after acute myocardial infarction(Virginia Commonwealth University Anakinra Remodeling Trial [VCU-ART] Pilot study). Am J Cardiol 105: 1371-1377, 2010.

33. Projahn D and Koenen RR: Platelets: key players in vascular inflammation. J Leukoc Biol 92: 1167-1175, 2012.

34. van den Akker F, Deddens JC, Doevendans PA and Sluijter JP: Cardiac stem cell therapy to modulate inflammation upon myocardial infarction. Biochim Biophys Acta 1830: 2449-2458, 2013. 